\title{
Isolated "puffy hands" following COVID-19: clue to a long-term capillary leakage syndrome?
}

\author{
Jacopo Ciaffi ${ }^{1}$ - Riccardo Meliconi ${ }^{1,2}$ - Piero Ruscitti ${ }^{3} \cdot$ Annamaria lagnocco $^{4} \cdot$ Clodoveo Ferri $^{5,6}$. \\ Roberto Giacomelli ${ }^{7}$. Francesco Ursini ${ }^{1,2}$
}

Received: 16 June 2021 / Revised: 17 June 2021 / Accepted: 19 June 2021 / Published online: 25 June 2021

(c) International League of Associations for Rheumatology (ILAR) 2021

\section{Dear Editor,}

Post-acute sequelae of SARS-CoV-2 infection (post-acute COVID-19 syndrome (PACS)) represent a novel challenge for physicians dealing with individuals recovering from COVID-19. However, the entire spectrum of long-term consequences of COVID-19 is still far to be fully elucidated [1]. Although persistent cutaneous and musculoskeletal complaints have been described in long-hauler patients [2], literature about the manifestations of potential rheumatological interest developing weeks to months after COVID-19 is still limited.

Here we share the cases of two female patients aged, respectively, 28 and 33 years, attending our rheumatology clinic between March and April 2021 with a history of recent-onset, painful, bilateral swelling of the hands. At the time of the first evaluation, the patients reported no other

Jacopo Ciaffi

jacopo.ciaffi91@gmail.com

1 Rheumatology Unit, IRCCS Istituto Ortopedico Rizzoli, SSD Medicina e Reumatologia, 40136 Bologna, Italy

2 Department of Biomedical and Neuromotor Sciences (DIBINEM), Alma Mater Studiorum University of Bologna, Bologna, Italy

3 Rheumatology Unit, Department of Biotechnological and Applied Clinical Sciences, University of L'Aquila, L'Aquila, Italy

4 Academic Rheumatology Centre MFRU, Dipartimento Scienze Cliniche E Biologiche, Università Degli Studi Di Torino, Torino, Italy

5 Rheumatology Unit, School of Medicine, University of Modena and Reggio Emilia, Modena, Italy

6 Rheumatology Unit, Rheumatology Hospital "Madonna Dello Scoglio", Cotronei, Crotone, Italy

7 Unit of Allergology, Immunology, Rheumatology, Department of Medicine, Università Campus Bio-Medico Di Roma, Rome, Italy medical conditions and in particular no history of symptoms consistent with Raynaud's phenomenon, were not taking any medication and had no known allergies.

Both patients reported a positive SARS-CoV-2 real-time PCR on nasopharyngeal swab, respectively, 6 and 8 weeks before the onset of symptoms. The course of COVID-19 was mild, characterized by headache and myalgia in one case and dry cough with low-grade fever in the other. Notably, hand swelling was not present during the acute phase of the infection. After resolution of symptoms, a second nasopharyngeal swab was obtained, testing negative.

Clinical examination revealed a moderate bilateral, nonpitting oedema of the hands and fingers, with erythematous skin, fissures over the interphalangeal joints and tenderness on palpation (patient 1, Fig. 1A). Skin thickening, fingertip lesions and telangiectasia were absent; no urticarial eruptions or pernio was present. Radial and brachial pulses were symmetrical.

Complete blood count, liver, kidney and thyroid function were normal, as were acute phase reactants and levels of complement fractions. Furthermore, antinuclear antibodies, antineutrophil cytoplasmic antibodies, cryoglobulins, anticyclic citrullinated peptide antibodies and rheumatoid factor were absent.

Nailfold videocapillaroscopy was performed in both patients, demonstrating mild pericapillary oedema but wellpreserved capillary distribution, arrangement and morphology (patient 1, Fig. 1B). Ultrasound of hands and wrists confirmed the oedematous thickening of subcutaneous layer with no evidence of synovitis, joint effusion or tenosynovitis. Finally, arterial and venous Doppler sonography of the upper limbs was normal. Empirically, a short course of glucocorticoids was prescribed, resulting in only minor improvement.

Besides the well-known dermatological sequelae of COVID-19 [2], our cases highlight the possibility of a 
Fig. 1 A Moderate bilateral, non-pitting oedema of the hands and fingers, with erythematous skin and fissures over the interphalangeal joints. B Mild pericapillary oedema but wellpreserved capillary distribution, arrangement and morphology

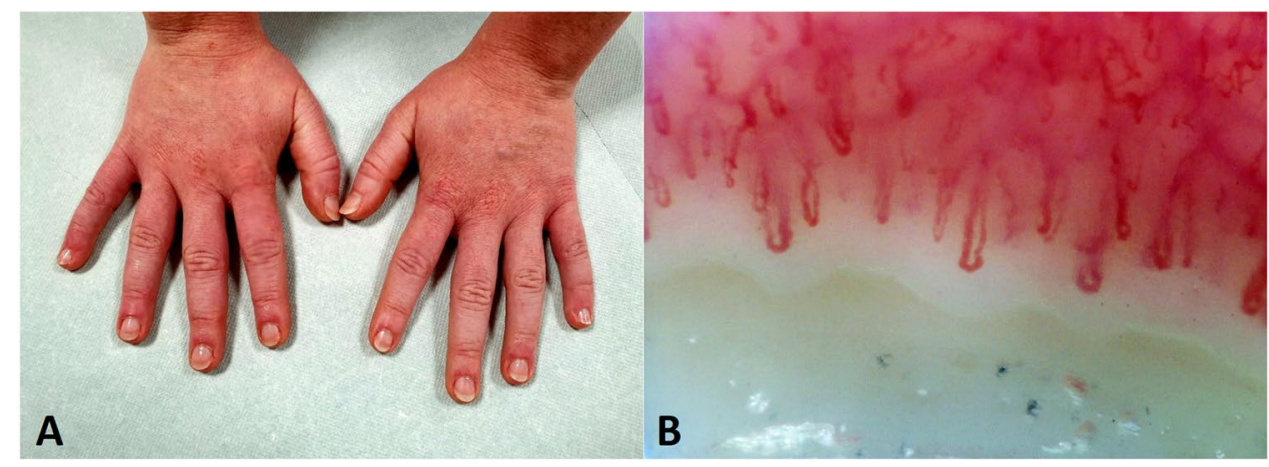

potential novel manifestation of interest for the rheumatologist, developing weeks after the acute infection.

Although a definitive causal relationship between SARSCoV-2 and development of cutaneous symptoms cannot be confirmed, the close temporal association with COVID-19, the atypical presentation and the absence of clinical data pointing to a clear diagnosis of connective tissue - very early systemic sclerosis in particular - or vascular disorders, narrow the differential diagnosis.

The pathophysiology of PACS has not been clarified, but findings from acute COVID-19 patients provide interesting clues to a potential mechanism. Capillary dysfunction and microvascular damage have been shown in acute COVID19 patients [3], with increased permeability and subsequent leakage [4]. Further, acro-ischaemic lesions were found to be common during the course of SARS-CoV-2 infection [5], and signs of endothelial activation have been histologically described in acral lesions obtained from COVID-19 patients [6]. Therefore, on the basis of this background, we feel that a long-term endothelial impairment resulting in acral capillary leakage may explain the clinical presentation observed in the two described cases, suggesting that the development of manifestations mimicking the puffy hands seen in early systemic sclerosis may be expected even weeks after COVID19. If confirmed, this "capillary permeability hypothesis" may represent a fascinating mechanism to be explored in explaining other organ-specific manifestations of the PACS galaxy, including neuropsychiatric, renal or gastrointestinal complaints.

\section{Declarations}

Consent for publication Patient's written consent for publication was obtained.

Disclosures None.

\section{References}

1. Nalbandian A, Sehgal K, Gupta A, Madhavan MV, McGroder C, Stevens JS et al (2021) Post-acute COVID-19 syndrome. Nat Med 27(4):601-615

2. McMahon DE, Gallman AE, Hruza GJ, Rosenbach M, Lipoff JB, Desai SR et al (2021) Long COVID in the skin: a registry analysis of COVID-19 dermatological duration. Lancet Infect Dis 21(3):313-314

3. Østergaard L (2021) SARS CoV-2 related microvascular damage and symptoms during and after COVID-19: consequences of capillary transit-time changes, tissue hypoxia and inflammation. Physiol Rep 9(3):e14726

4. Teuwen LA, Geldhof V, Pasut A, Carmeliet P (2020) COVID-19: the vasculature unleashed. Nat Rev Immunol 20(7):389-391

5. Alonso MN, Mata-Forte T, García-León N, Vullo PA, RamirezOlivencia G, Estébanez M et al (2020) Incidence, characteristics, laboratory findings and outcomes in acro-ischemia in COVID-19 patients. Vasc Health Risk Manag 16:467-478

6. Recalcati S, Barbagallo T, Frasin LA, Prestinari F, Cogliardi A, Provero MC et al (2020) Acral cutaneous lesions in the time of COVID-19. J Eur Acad Dermatol Venereol 34(8):e346-e347

Publisher's note Springer Nature remains neutral with regard to jurisdictional claims in published maps and institutional affiliations. 